\title{
The Incidence of the Third Trimester Complications in Gestational Diabetes in our Hospital
}

\author{
Migena PRIFTI, MD ${ }^{1}$, Arben HAXHIHYSENI, MD, PhD $^{2}$ \\ ${ }^{1}$ Department of Obstetrics - Gynecology, Regional Hospital Durres, Albania \\ ${ }^{2}$ Clinical head of Gynecology' department; Regional Hospital Durres, Albania
}

\begin{abstract}
Introduction: The increasing incidence of gestational diabetes is an important topic for the public health. Sedentary lifestyle is strongly associated with this pathology, while gestational diabetes is an important risk factor for fetal complications and complications during childbirth also. Aim: The incidence of gestational diabetes complications in third trimester in our hospital. Methodology: A double blind study was performed based in medical recording of the pregnant women ultrasound exam, value of the fasting plasma glucose and the oral glucose tolerance test OGTT, measured in the risk group. This study included 5019 pregnant women hospitalized in Regional Hospital of Durres during the period May 2012 till November 2013, and 57 of them were treated for problems related to gestational diabetes. Results: Premature birth (under 37 weeks) occurred in 138 women, 34 of those were diagnosed with GD. 159 cases were diagnosed with preeclampsia and 10 patients also were with GD. 30 women were diagnosed with fetal hypotrophy and five of them were also with GD. Urinary infection in GD patients was diagnosed in 110 women.
\end{abstract}

Keywords: Gestational Diabetes, fasting plasma glucose FPG, pregnancy complications, third trimester.

\section{Introduction}

Gestational diabetes (GD) in pregnancy is defined as glucose intolerance. In pregnancy, the incidence rate is 2 to $4 \%$ but global guidelines show that this index is related with risk factor and in the Mediterranean area (such as our country), the incidence value is between 3 to $5 \% .(1,2$,$) . The$ differences between the diagnose protocol criteria of our country with the others make difficult the met analyze. Also the relation between gestational diabetes prevalence and demographic factor is not very clear. We can say that ethnicity is an independent risk factor for GDM. In 2009, the term of Prediabetes was introduced in our protocol of treatment including all cases with fasting plasma glucose between $95 \mathrm{mg} / \mathrm{dl}$ and $110 \mathrm{mg} / \mathrm{dl} .(3,4)$. In prediabetes the patients has no somatic concerns or complains, nor problems linked to obesity. The prediabetes is used to highlight the group risk for developing diabetes disease. Cases with prediabetes has impaired tolerance for glucose (IGT)

Risk factors for GD $(5,6,7)$

- Ethnicity (Hispanic, afro-Americana or Asiatic)

- Woman age up to 30 year old

- Diabetes insulin dependent in family

- Obesity

- Recurrent urinary infections or genital infections

- Multi parity

- Missed abortion, fetus mort in uterus and fetal weight up to 4000 gram.

- The presence of glucosuria

- Microcosmic ovaries syndrome

Studies shows that GD is diagnosed in $4.8 \%$ of woman without risk factors ( 8 )

\section{Methodology}

This is a prospective study, performed on the pregnant patients in the Regional Hospital of Durres. During 18 month of study (May 2012 until November 2013) we collected also data from the medical records for the complications in the third trimester of pregnancy. To perform this study we use plasma glucose values measured Also we use the fasting glucose test, oral glucose tolerance test (50gr of sugar) and $\mathrm{HbA} 1 \mathrm{C}$ test to confirm diagnose. Obstetrical history and personal history were also useful.

\section{The population}

The patients were randomly selected during the routine control and patients hospitalized in pregnancy related disorders service PDS. 5019 cases were included in our study and from them we choose the risk group. Interpretation is based on venous plasma glucose results before and 2 hours after a $75 \mathrm{~g}$ oral glucose load.

\begin{tabular}{|c|c|c|c|}
\multicolumn{3}{c}{ OGTT } \\
\hline No diabetes & $\geq 6.0$ & $<7.8$ & $\begin{array}{c}\text { No excess micro- nor macro- } \\
\text { vascular risk }\end{array}$ \\
\hline Prediabetes & $6.1-6.9$ & $7.8-11.0$ & $\begin{array}{c}\text { Excess macro- but not micro- } \\
\text { vascular risk }\end{array}$ \\
\hline Diabetes & $\geq 7.0$ & $\geq 1.1$ & $\begin{array}{c}\text { Excess macro- and micro- } \\
\text { vascular risk }\end{array}$ \\
\hline
\end{tabular}

The treatment protocol of gestational diabetes in third trimester

We use the existing protocol for the gestational diabetes $(9,10,11)$ in the third trimester:

1) The bio and physical profile: the fetal weight and the position of the fetus

2) Cardiotocography- monitoring of fetal cardiac activity

3) Biochemical examinations

4) Measure the weight and the blood pressure of the patient.

5) Control of fasting plasma glucose and oral glucose tolerance test (50gr of sugar) in $23^{\text {th }}-25^{\text {th }}$ week of the pregnancy 


\section{International Journal of Science and Research (IJSR) \\ ISSN (Online): 2319-7064}

Index Copernicus Value (2013): 6.14 | Impact Factor (2014): 5.611

The complications of the third trimester:

1) Urinary infections and genital infections (these infections might begin in the first trimester) (12)

2) Another complications related to diabetes is preeclampsia. $(13,14)$ The preeclampsia is common at woman with diabetes $(12 \%)$. Chronic hypertension is another complication that occurs in 1 to 10 diabetic women. The women that have chronic hypertension and diabetes have also high risk to develop fetal hypotrophy, preeclampsia, placental abruption and maternal maligned hypertension.

3) Fetal hypotrophy $(15,16)$; Changes of the liquid amount ( polyhydramnios and oligohydramnios) $(17,18)$.

4) Premature partum(,19,20, 21)

5) Fetal macrosomia(22). All babies born from hyperglycemia moms have double odds for trauma during the partum. Odds for section cesarean in this case are triple comparing with healthy pregnant women. Babies born from hyperglycemia moms also are risked to recover in the Neonatal Intensive Care Unit for neonatal problems. That's why is necessary to prevent neonatal problems by monitoring the fetal weight.

6) Feto-morto in utero

\section{Results}

From all the cases (5019) in our hospital during the time of study, 1000 of them had shown complications during the third trimester. 490 patients out of these 1000, were hospitalized in the PDS of our hospital. In total, 98 patients were diagnosed with pre-diabetes and 5 patients with diabetes type 2 , insulin dependent.

1) According to the studies we've been referring to, we see increasing proportions of gestational diabetes with urinary infections and genital infections up to $27.3 \%$. In our study we have 10 cases with recurrent urinary infection with FPG $>100 \mathrm{mg} / \mathrm{dl}$ in 100 patients treated for urinary infections. Soo the results for this complication in gestational diabetes is $10 \%$.

2) Preeclampsia. Its incidence at women with GDM is about $12 \%$. (23,). The odds for preeclampsia are positively correlated with the age of the woman and the level of glicemia. Results from another study have shown: when early glucose was $<105 \mathrm{mg} / \mathrm{dL}$ the rate of preeclampsia was $7,8 \%$ but for early glucose level $>105$ $\mathrm{mg} / \mathrm{dL}$ preeclampsia rate was $13,8 \%$.(24).Also, from the same study, body mass index in pregnancy is related with preeclampsia. In our hospital, 159 cases were treated for preeclampsia, and only 10 of them $(6.2 \%$ in total $)$ had high values of glucose.
3) Fetal hypotrophy. Only 5 cases are registred with fetal hypotrophy and there in the pregnancy more the 41 weeks. Changes of the liquid amount (polyhydramnios and oligohydramnios ) : From 7 cases who had polyhidramnios, five of them arrive until the 39 week of pregnancy. From the beginning of the week 32th, 5-7 \% of cases had shown placenta calcification and oligohydramnios.

4) Premature partum is the most important complication to gestational diabetes. Some studies show the correlation between the DG and the premature partum is about $6.2 \%$ $(, 25,26,27)$. In our study we have 34 of all the cases with premature partum, 18 of them were in toccolytic therapy and the delivery was at the 37 th week. 5 cases of them had premature partum at the 35- 37 week of pregnancy. Only one patient had a missed abortion in the $8^{\text {th }}$ week cause of the hypertension(values $150 / 100 \mathrm{~mm} / \mathrm{Hg}$ ), 10 cases had incomplete abortion. The patients with GD, who had a preterm labor, are $10 \%$ to cases hospitalized for premature partum in our hospital.

5) Fetal macrosomia. All babies born from hyperglycemia moms have double odds for trauma during the partum. Odds for section cesarean are triple comparing with healthy pregnant women. Babies born from hyperglycemia moms also are risked to recover in the Neonatal Reanimation Department for neonatal problems. That's why itis necessary to prevent neonatal problems by monitoring the fetal weight. In our study 217 newborns weighted over 4000 grams.

6) During the time of study feto- morto in utero from gestational diabetes resulted zero.

The complications that come from GD during the third trimester of pregnancy are 11\% (in total)

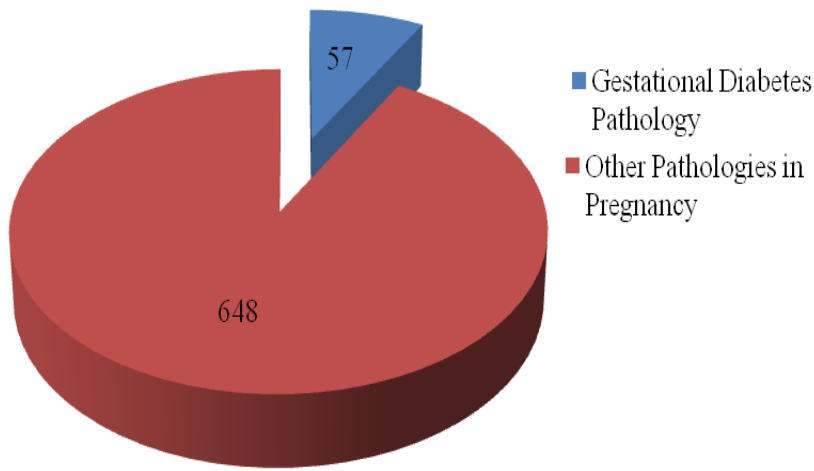

Figure 1: Present the cases with diabetes and the proportions of pregnancy hospitalized in the pathology department

Other pathology - 648 cases; GD pathology - 57 cases 
International Journal of Science and Research (IJSR)

ISSN (Online): 2319-7064

Index Copernicus Value (2013): 6.14 | Impact Factor (2014): 5.611

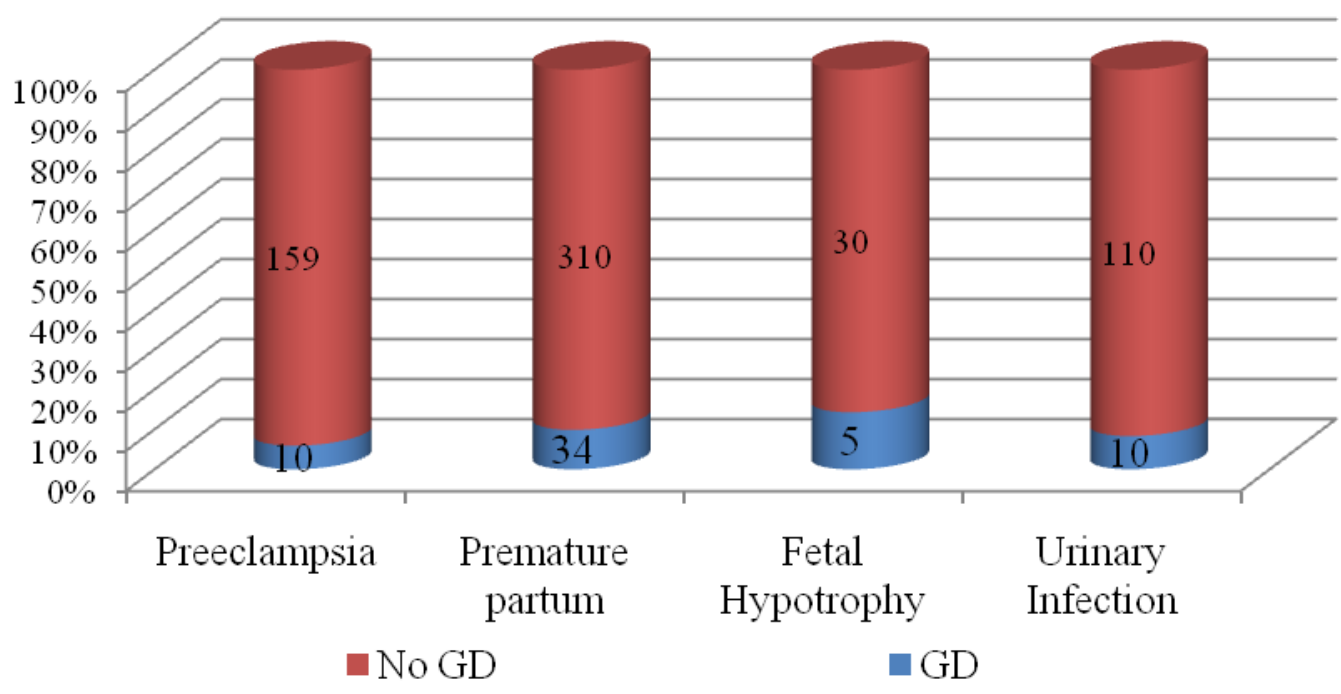

Figure 2: Differences between complications in the third trimester in gestational diabetes patients comparing with non gestational diabetes patients

\section{Discussion}

According to our data and referring to recent studies we conclude:

- Preeclampsia is associated with gestational diabetes in 12 $\%$, but in our study we conclude that the rate is lower to $6,2 \%$. This rate difference may be depending by geographic position and demographic characteristics, the exact time of examination and diagnose, and the interpretation of OGTT and clinical signs.

- Other reason we can mention may be the lack of information for the diabetes examinations during the pregnancy. In relation with gestational diabetes and premature partum we don't have correct data because same premature partum are associated also with the fetus weight. Thisis more common in macrosomia babies or in woman that has irregular menstrual cycle. In results $27 \%$ to our study that is not included. As a result we can say, a good monitored pregnancy infect directly in the outcome.

- The evaluation of placenta calcification by ultrasound, usually is not accompanied with glicemia test and this way the correct diagnose is a bit difficult. For this reason the results for deseases to amniotic fluid and placenta are not signed corretly in this study.

- The results of this study are relatives because not all the patients made their visit and laboratory examination correctly the age of pregnancy.(only $12 \%$ of them).

\section{Conclusion}

- It's important to mention that in our country GD as pathology isn't very common. The same is also for its complications in pregnancy. That's why we should have GD in focus. The information is very important not only in the risk group but also in the population. This way we can prevent the gestational diabetes complications.

- Is necessary a specific protocol for measure the fasting glycemia and OGTT (75gr of sugar) in 22th-26th week of the pregnancy.

\section{References}

[1] Hunt KJ , Schuller KL: the increasing prevalence of diabetes in pregnancy 2007

[2] Brown Diabetes Testing First Prenatal Visit Universal Rec. Medscape Medical News [serial online]. November 7, 2013, accessed January 12, 2013.

[3] Diagnosing diabetes and learning about prediabetes :American Diabetes Association

[4] Guideline for diabetes in pregnancy -joslin diabetes center

[5] Spong CY, Guillermo L, Kuboshige J, T. Cabalum Recurrence of gestational diabetes mellitus: Identification of Risk Factors. Am J Perinatol. January 1998, 15 (1): 29-33. [Medline].

[6] Moses R, Griffiths R, Davis W. Gestational diabetes: Women shouldnt EU are all tested?. Aust N Z J Obst Gynaecol. November 1995, 35 (4): 387-9. [Medline].

[7] Tobias KC, Hu FB, Forman JP, Chavarro J, Zhang C. Increase Risk of Gestational Diabetes Mellitus After hypertension: Findings from a Large Possible study team. Diabetes Care. July 2011,

[8] Blumer I, Hadar, Hadden DR, et al. Diabetes and pregnancy: an endocrine Society clinical practice guideline. J Clin Endocrinol metab. November 2013, 98 (11): 4227-49. [Medline].

[9] Guideline American Diabetes Association. Standards of medical care in diabetes - 2010. Diabetes Care. January 2010, 33 Suppl 1: S11 - 61. [Medline]. [Full text]. American Diabetes Association. Standards of medical care in diabetes-2013. Diabetes Care. January 2013, 36 Suppl 1: S11 - S66. [Full Text]

[10] Metzger BE, Coustan Dr.Summary and recomandation of workshop conference on gestacional diabetes mellitus. Diabtes care 1998

[11]ACOG Practice Bulletin. Clinical management guidelines for obstetrician - gynecologists. Number 30, September, 2001 (replaces Technical Bulletin Number 200, January 1994).

[12] Spong CY, Guillermo L, Kuboshige J, T. Cabalum Recurrence of gestational diabetes mellitus: 


\section{International Journal of Science and Research (IJSR) \\ ISSN (Online): 2319-7064}

Index Copernicus Value (2013): 6.14 | Impact Factor (2014): 5.611

Identification of Risk Factors. Am J Perinatol. January 1998, 15 (1): 29-33. [Medline].

[13] Hedderson MM, Ferrara A. : High Blood Pressure Before and up during Early Pregnancy is Associated with an increased risk of gestational diabetes mellitus. Diabetes Care. December 2008,

[14] Tobias KC, Hu FB, Forman JP, Chavarro J, Zhang C. Increase Risk of Gestational Diabetes Mellitus After hypertension: Findings from a Large Possible study team. Diabetes Care. July 2011,

[15] Gestacional diabetes IDDT

[16]Royal College of obstetricians and gynecologist :Shoulder dystocia PDF

[17] S.Hebbar 2011 : Reference range of amniotic fluid index in late third trimester

[18]G. Desoye 2007 : the human placenta in GDM

[19] Pregnancy EN RTP -2 PDF-international diabetes federation 2009

[20] Tongsong T, W Piyamongkol, Sreshthaputra O. : The Accuracy of ultrasonic fetal weight: a Comparison of Three equations Employed for Evaluation of fetal weight. J Med Assoc Thai. July 1994,

[21]Benacerof BR, Gelman R , Frigoletto FD : Sonographically values fetal weights November 1988

[22] Bernstein IM, Catalano PM. Influence of fetal luck on the ultrasound estimation of fetal weight in diabetic mothers. Obst Gynecol. April 1992,.

[23] Ines Mrizak et al :Gestational hypertension and preeclampsia Journal of hypertension december 2004

[24] Hadar Eran , Hod Moshe : Maternal complication of GDM - Other types of diabetes mellitus $13 / 08 / 2014$

[25] Heenry L. Davila MD MPH ,Maris L.Pena MD MPH Zaskia Matos : Clinical and Epidemiological Profile of Diabetes Mellitus in Pregnancy, 2011

[26] Jacques Leperco MD , J.Coste MD et al : Factors Associated With Preterm Delivery in Women with GDM (Diabetes Care, 2004)

[27] Bar-Hava I , Barnard Y et al : Gestational diabetes and preterm labour 1997 Jun

[28] Marlene Busco \& al: Gestational Diabetes Testing in 24 Weeks. Medscape Medical News. May 29, 2013. 